\title{
Proposta de classificação de vegetais considerando características nutricionais, sensoriais e de técnicas de preparação ${ }^{1}$
}

\author{
Proposal of vegetable classification considering \\ nutritional and sensory characteristics \\ and preparation techniques
}

Lúcia Chaise BORJES²

Suzi Barletto CAVALLI ${ }^{3}$

Rossana Pacheco da Costa PROENÇA ${ }^{2,3}$

\section{R E S U M O}

Apresenta-se uma classificação de vegetais utilizados no processo produtivo de refeições comerciais e coletivas, correlacionando concomitantemente as características nutricionais, sensoriais e técnicas de processamento. Esta classificação foi concebida para utilização inicial no sistema de Avaliação da Qualidade Nutricional e Sensorial, desenvolvido para ser utilizado em paralelo ao Análise de Perigos e Pontos Críticos de Controle, visando garantir a qualidade nutricional e sensorial de refeições. A pesquisa iniciou-se com a identificação das classificações de vegetais disponíveis na literatura científica e sua análise, verificando a possível adequação às necessidades do sistema Avaliação da Qualidade Nutricional e Sensorial. Como nenhuma das classificações apresentou as características necessárias, partiu-se para a concepção de uma nova classificação de vegetais, denominada Classificação de Vegetais Avaliação da Qualidade Nutricional e Sensorial. Para tanto, foram sendo realizados cruzamentos entre as características identificadas nas classificações, até associar simultaneamente as características desejadas. A nova classificação resultou da associação entre a classificação pela parte botânica comestível e a classificação segundo o teor de carboidratos, considerando-se, também, as técnicas de preparação. Como resultado, foram analisados 99 vegetais, divididos em sete grupos. Evidencia-se que o controle da interação entre aspectos nutricionais, sensoriais e de técnicas de processamento desde a aquisição até a distribuição do alimento pronto poderá, potencialmente, resultar em alimentos mais saudáveis. Além disso, o controle da qualidade sensorial dos alimentos pode influenciar a escolha e consumo dos alimentos preparados.

Termos de indexação: Classificação. Conservação de alimentos. Normas da qualidade de alimentos. Proteínas vegetais. Vegetais. Tecnologia de alimentos.

\footnotetext{
1 Artigo elaborado a partir da dissertação de L.C. BORJES, intutulada "Concepção da classificação de vegetais para a aplicação no sistema de avaliação da qualidade nutricional e sensorial - AQNS”. Universidade Federal de Santa Catarina; 2007. Apoio: Coordenação de Aperfeiçoamento de Pessoal de Nível Superior e Conselho Nacional de Desenvolvimento Científico e Tecnlógico - Edital Universal/2004 - Processo 470613/2004-1.

2 Universidade Federal de Santa Catarina, Programa de Pós-Graduação em Nutrição, Núcleo de Pesquisa de Nutrição em Produção de Refeições. Caixa Postal 476, Campus Universitário, Trindade, 88040-900, Florianópolis, SC, Brasil. Correspondência para/Correspondence to: L.C. BORJES. E-mail: <lborjes@gmail.com>.

3 Universidade Federal de Santa Catarina, Centro de Ciências da Saúde, Curso de Nutrição, Departamento de Nutrição. Florianópolis, SC, Brasil.
} 
646 | L.C. BORJES et al.

\section{A B S T R A C T}

This paper presents a classification of vegetables used in foodservices and large-scale meal production, concomitantly correlating nutritional and sensory characteristics and processing techniques. This classification was conceived to be used initially in the Nutritional and Sensory Quality Assessment and together with the Hazard Analysis and Critical Control Points, in order to guarantee the nutritional and sensory quality of meals. The research began by identifying the vegetable classifications available in the literature and their analysis, verifying if it is possible to adjust them to the requirements of the Nutritional and Sensory Quality Assessment system. As none of the classifications presented the necessary characteristics, a new classification of vegetables was conceived and named Nutritional and Sensory Quality Assessment Vegetable Classification. For this purpose, the characteristics identified in the classifications were crossed until the desired characteristics were simultaneously associated. Thus, the new classification resulted from the association between the classifications of the edible plant part and the carbohydrate content, in addition to the preparation techniques. A total of 99 vegetables were then analyzed and divided into 7 groups. Controlling the interaction between nutritional and sensory aspects and processing techniques, from the purchase to the distribution of the foods, can result in healthier meals. Furthermore, controlling the sensory quality of the foods can influence the selection and consumption of the prepared foods.

Indexing terms: Classification. Food preservation. Food quality standards. Vegetables. Food technology.

\section{N T R O D U ÇÃ O}

Em 1996, os Estados Unidos e a Comunidade Europeia lançaram o Rome Declaration on World Food Security and World Food Summit Plan of Action com o compromisso comum de atingir a segurança alimentar para todos ${ }^{1}$. Mesmo antes dessa declaração, no Brasil, o conceito de segurança alimentar já englobava a quantidade de produção e acesso aos alimentos; as doenças de origem alimentar e nutricional; bem como a qualidade dos alimentos: nutricional, físico-química e microbiológica. Outros aspectos ressaltados são os cuidados com relação às técnicas de produção para a garantia da qualidade nutricional e os efeitos do tratamento culinário sobre o alimento. O País adotou esse conceito a partir de 1986, o qual se consolidou em 1994 com a realização da I Conferência Nacional de Segurança Alimentar².

Em 2004, a Organização Mundial da Saúde adotou a Global Strategy on Diet, Physical Activity and Health, a qual, com relação à nutrição, recomenda uma dieta saudável. Essas recomendações devem servir como referência para elaboração de políticas públicas, levada em consideração a situação do local ${ }^{3}$.

Em 2006, com a sanção da Lei Orgânica de Segurança Alimentar e Nutricional (LOSAN), há a criação do Sistema Nacional de Segurança Alimentar e Nutricional (SISAN), com o objetivo de assegurar o direito humano à alimentação adequada, o qual reforça o conceito de segurança alimentar e nutricional já adotado no Brasil ${ }^{4}$.

Nesse sentido, o controle de qualidade do alimento não se dá unicamente pela inocuidade, mas pelas formas de processamento desse alimento, que podem comprometer as suas qualidades naturais, afetando o princípio de uma alimentação saudável ${ }^{5}$.

Seguindo essa lógica, a qualidade na produção de refeições pode ser implementada por meio de metodologias que visem ao controle dos processos de produção de alimentos seguros e sensorialmente adequados. Assim, a utilização de técnicas de manipulação e processamento, bem como o equilíbrio entre os componentes são fundamentais. Além desses, a preocupação com a apresentação final começa com os cuidados durante o armazenamento, pré-preparo, preparo, espera e distribuição desses alimentos. Para a gestão de uma Unidade Produtora de Refeições (UPR), torna-se necessário cumprir requisitos, tais como: os técnicos, os operacionais, os sanitários e os culinários com o objetivo de atingir os resultados esperados 5 .

Nesse contexto, foi desenvolvido o sistema de Avaliação da Qualidade Nutricional e Sensorial 
(AQNS) que busca o controle do processo produtivo de refeições para monitoramento da qualidade nutricional e sensorial dos alimentos. O AQNS foi desenvolvido para ser utilizado em paralelo à Análise de Perigos e Pontos Críticos de Controle (APPCC) que visa ao controle higiênico-sanitário. Para a utilização do AQNS, é necessária a definição dos critérios nutricionais e sensoriais para os diferentes grupos de alimentos preparados para compor uma refeição ${ }^{6}$.

A qualidade nutricional pode ser definida como a capacidade de um alimento em atender um indivíduo com os nutrientes necessários para sua manutenção, desenvolvimento e funcionamento orgânico normal ${ }^{7}$.

O conceito de qualidade sensorial engloba um conjunto de características que são percebidas pelos cinco sentidos, ao se degustar um alimento, quais sejam: aspecto, cor, sabor, aroma e consistência. Os parâmetros relativos aos sentidos podem ser identificados em microestruturas do alimento e levar a ações estimulantes ou inibitórias percebidas por vários fatores metabólicos e sensitivos $^{8}$. A qualidade sensorial de um alimento relaciona-se, ao mesmo tempo, com o alimento e com as características fisiológicas, psicológicas e sociológicas do indivíduo que o avalia ${ }^{9}$.

O processo produtivo de refeições em uma Unidade Produtora de Refeição (UPR) consiste nas etapas de planejamento do cardápio, aquisição dos gêneros necessários, recebimento e armazenamento de acordo com a característica de cada produto, pré-preparo dos gêneros (higienização, descasque, fracionamento e mistura), preparo (tratamento térmico), espera para distribuição, distribuiçã̃o e destino de sobras e restos ${ }^{10}$.

Complementando essa ideia, com um planejamento competente, um conhecimento aprofundado dos processos executados e a disseminação do conceito de alimentação saudável, a UPR estará visando à melhoria dos serviços prestados ${ }^{11}$.

Destaca-se que a lógica de implantação do sistema APPCC em UPR determina que, na etapa de elaboração dos fluxogramas, há a necessidade de agrupamento das receitas considerando as igualdades ou semelhanças, tanto de matérias-primas quanto de etapas de processamento ${ }^{12}$. Assim, para a implantação e aplicabilidade do sistema APPCC em uma UPR, os alimentos são agrupados pelas características semelhantes com relação às possibilidades de contaminação. No sistema AQNS, o processo é semelhante, havendo a necessidade de formar grupos dos diferentes alimentos utilizados na preparação de refeições, buscando a interação entre seus aspectos nutricionais, seus aspectos sensoriais e suas técnicas de processamento, conforme esquematizado na Figura 1.

Caso esse agrupamento não possa ser determinado, tanto no APPCC quanto no AQNS, a especificação dos pontos críticos de controle, para posterior monitoramento, não será possível, porque em nenhum dos sistemas se trabalha com o alimento sozinho. Como se busca trabalhar com conjunto de critérios que atendam a um determinado grupo de alimentos, se esse agrupamento não for realizado, teremos um conjunto de critérios para cada alimento ou preparação, tornando os sistemas praticamente inviáveis de serem aplicados no processo produtivo de refeições. Nesse caso, como o sistema funciona a partir da realização de medições e observações do fluxo produtivo das refeições, se essas atividades tiverem que obedecer a critérios específicos para cada alimento, a quantidade de documentos e monitora-

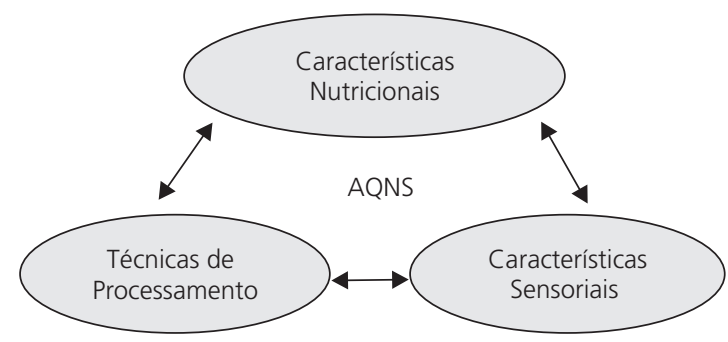

Figura 1. Esquema das necessidades de interação de características nutricionais, sensoriais e de técnicas de processamento para o desenvolvimento de critérios e aplicação do sistema Avaliação da Qualidade Nutricional e Sensorial de refeições (AQNS). 
mentos certamente atrapalhará o andamento do processo.

Salienta-se que a recomendação com relação aos processos de garantia de qualidade é no sentido de que os procedimentos de monitoramento e controle sejam incorporados às atividades diárias ${ }^{13}$. Na gestão da produção de refeições, nessa mesma lógica, os sistemas APPCC e AQNS devem ser estruturados de maneira que seja possível realizá-los como parte da rotina da UPR.

Esse estudo objetivou conceber uma classificação de vegetais que se adequasse às necessidades do sistema AQNS, associando as características nutricionais, as características sensoriais e as características de técnicas de processamento em refeições.

\section{M É T O D O S}

Foi realizada um levantamento bibliográfico no banco de dados PubMed, da National Library of Medicine, na Scientific Electronic Library Online (SCIELO) e na biblioteca da Universidade Federal de Santa Catarina, utilizando-se os seguintes descritores: vegetables classification, vegetables, vegetables and nutritional quality, vegetables and sensorial quality e vegetables and food processing.

Identificaram-se, então, as seguintes classificações de vegetais: classificação botânica e pela família; classificação de vegetais pela parte botânica; classificação de vegetais pela cor e pigmentos; classificação de vegetais segundo teor de carboidratos.

Considerando-se um conjunto de 99 vegetais utilizados na produção de refeições no Brasil, foi confeccionado um quadro, conforme exemplo constante no Quadro 1 para Abóboras, contendo as seguintes colunas: nome botânico, família ${ }^{14-17}$, parte botânica ${ }^{10,18}$, teor de carboidratos ${ }^{10,19}$, pigmentos e classificação pela cor r $^{10,20,21}$.

Empregando a ferramenta de classificação de texto de tabela do programa Word 2000, experimentaram-se todas as possibilidades de cruzamento entre as colunas, com relação aos diferentes vegetais. Após várias tentativas, chegou-se a uma classificação considerada adequada à aplicação do sistema AQNS, que será exposta.

\section{RESULTADOS E DISCUSS ÃO}

Vegetal (do latim vegetale) é relativo ou procedente de plantas. De acordo com o Codex Alimentarius, a categoria de vegetais inclui também: cogumelos e fungos, raízes e tubérculos, grãos e legumes, aloe vera, algas, nozes e sementes $^{22}$. Conforme o Guia Alimentar Americano, vegetais podem ser crus ou cozidos, frescos, congelados, enlatados ou desidratados; e pode ser inteiro, picado ou triturado ${ }^{23}$. Em se tratando de alimentos, esse termo se torna bastante genérico, por isso alguns autores utilizam o termo hortaliça para designar os vegetais cultivados em hortas ou como nome genérico das verduras e legumes. Verduras e legumes, por sua vez, são definidos como a parte comestível das plantas ${ }^{10}$.

Classificação é o ato de classificar, distribuindo em classes e/ou grupos segundo sistema ou método pré-definido ${ }^{24}$. Os sistemas de classificações de vegetais encontrados na literatura e a sua análise para auxiliar a atingir os objetivos propostos neste estudo são expostos a seguir.

\section{Classificação botânica e pela família}

A classificação botânica e pela família resulta em 17 grupos de vegetais ${ }^{14-17}$. Na sua análise, percebeu-se que não seria viável a sua aplicabilidade ao sistema AQNS, pois, como comentado, o número de formulários e medições seria muito grande para ser incorporado às rotinas das UPR, dificultando ou, mesmo, inviabilizando a utilização do sistema AQNS.

Além disso, algumas famílias contêm vegetais que não apresentam semelhanças no processo de produção de refeições, apresentando diferentes técnicas de preparo ou diferentes características para a mesma técnica de preparo. Um 
exemplo são as solanáceas, representadas por batata-inglesa, tomate e berinjela. Em termos de consistência, a batata apresenta características diferentes devido à sua estrutura química, apresentando um teor elevado de amido comparado aos outros dois vegetais. Já a berinjela e o tomate apresentam características semelhantes em sua estrutura química e podem ser preparados utilizando-se técnicas de preparo semelhantes com relação ao tempo e à temperatura de cocção ${ }^{25}$. Contudo, a utilização culinária é bastante diferenciada, haja vista a utilização do tomate como tempero e base de molhos bastante difundidos.

Outro exemplo são as umbelíferas, representadas por cenoura, aipo e batata-baroa. Apesar de estes vegetais apresentarem a vitamina $A$ como uma característica comum, existem muitas características distintas, entre elas o teor de carboidratos, principalmente o amido, presente na batata-baroa, mas praticamente ausente no aipo. Além disso, são diferentes os tipos de pigmentos e as estruturas vegetais que esses vegetais apresentam, pois o aipo é um caule e os outros dois são raízes. Com essas diferentes características, os processos de cocção são diversos, tanto com relação ao tempo e temperatura, quanto nas técnicas utilizadas. O aipo é utilizado em refogado, em sopas, cozidos, caldos e tortas, enquanto que a cenoura e a batata-baroa podem ser utilizadas em sopas, cozidas, refogadas e em purês. Destaca-se, ainda, a possibilidade de utilização do aipo e da cenoura crus, em contraposição à impossibilidade de utilização da batata-baroa crua ${ }^{26}$.

\section{Classificação de vegetais pela parte botânica}

Observando-se a classificação pela parte botânica, podemos identificar semelhanças quanto à forma de preparo dos diversos vegetais, devido a características da estrutura química do tecido vegetal. Por exemplo, o brócolis e a couve-flor, ambos flores, podem ser preparados da mesma maneira, isto é, cozidos com os talos para baixo para obter um abrandamento uniforme. Outro exemplo seriam as folhas, couve-manteiga, mostarda, repolho e acelga. Todos eles devem ser preparados cozidos em pouca água, com panela sem tampa, ou refogados pelo menor tempo possível. Entretanto, não houve associação em alguns componentes nutricionais, como é o caso das raízes e tubérculos, por exemplo, cenoura e batatas, que apresentam diferentes teores de carboidratos, principalmente de amido. Devido a essas diferenças, o tempo de cocção desses vegetais é diferente. Além disso, a cenoura é um vegetal rico em carotenoide e a batata contém antocianidina, o que exige diferentes cuidados na cocção. Então, como aspecto positivo dessa classificação, tem-se a questão da estrutura do tecido vegetal, que, sendo semelhante conforme a parte da planta, pode resultar em processamentos também semelhantes. Em contrapartida, a composição química de nutrientes tradicionais e não tradicionais apresenta aspectos distintos, apesar de a estrutura química do tecido vegetal ser semelhante.

\section{Classificação de vegetais pela cor e pigmentos}

Ao se analisar a classificação pela cor e pigmentos $21,24,27$ observou-se que esta também não atendeu às necessidades do sistema AQNS, pois muitos vegetais, embora com a mesma cor, apresentam características diferentes, principalmente nas formas de preparo. Tem-se, por exemplo, o grupo da cor roxa, representado por repolho-roxo, berinjela e alcachofra. Embora esses vegetais possuam os mesmos pigmentos, as suas estruturas vegetais são distintas, pois se apresentam como folhas, frutos e flores, respectivamente. Consequentemente, as suas formas de preparo, bem como as características sensoriais também serão distintas.

Outro exemplo de mesmo pigmento com estrutura vegetal diferente podem ser os vegetais de cor verde, grupo no qual também existem as flores (brócolis), folhas (couve, mostarda, espinafre) e frutos (abobrinha e pimentão verde). Já 
para a cor branca temos couve-flor, aipo ou salsão e aipim ou mandioca. Nesse caso, além da estrutura vegetal diferente, representada por flor, caule e raiz, respectivamente, a composição química, principalmente em amido, é muito distinta. Consequentemente, as características nutricionais e sensoriais serão distintas, bem como as técnicas de processamento. Analisando-se, por exemplo, o tempo de cocção desses vegetais, quais sejam: couve-flor $\left(8-16^{\prime}\right)$, aipo ou salsão $\left(20-25^{\prime}\right)$ e aipim ou mandioca $\left(30-35^{\prime}\right)$, pode-se identificar a dificuldade em colocá-los num mesmo grupo para definir critérios de qualidade nutricional e sensorial ${ }^{10}$.

Além dessas questões, a classificação dos vegetais pela cor e pigmentos pode variar de acordo com países e/ou regiões. Isso pode ocorrer, de um lado, pelas variações de cor causadas por espécies diferentes ou áreas de cultivo distintas, que poderão resultar em alimentos com nomes semeIhantes, mas cores diferentes. Por outro lado, considerando que a percepção de cor pode ter um componente cultural, dependente também dos modos de preparo tradicionais de determinados vegetais, esses podem ser classificados em grupos diferentes. Assim, por exemplo, o aipo, o alho-poró e o aspargo são classificados no grupo dos brancos no Brasil21 e no grupo dos verdes nos Estados Unidos ${ }^{27}$. Nesse sentido, essas razões justificam a não adequação da classificação de vegetais pela cor e pigmentos para utilização no sistema AQNS.

\section{Classificação de vegetais segundo teor de carboidratos}

A classificação de acordo com o teor de carboidratos disponível na literatura científica é a que consta no clássico livro brasileiro de Técnica Dietética ${ }^{10}$, sendo a mesma desde a primeira edição publicada em 1963. Entretanto, considerando-se a origem dessa classificação, discutida pessoalmente com a autora do livro, ela provavelmente originou-se de dados de composição de alimentos norte-americanos disponíveis na década de 1930. Além disso, destaca-se que os métodos de identificação de nutrientes do ponto de vista laboratorial evoluíram desde aquela época.

Por isso, para analisar a viabilidade atual dessa classificação, realizamos um exercício de atualização desta, considerando os dados brasileiros, disponíveis na Tabela Brasileira de Composição de Alimentos (TACO) ${ }^{19}$. Quando se comparou a classificação original ${ }^{10} \mathrm{com}$ o resultado da TACO - Tabela Brasileira de Composição de Alimentos, observou-se que a maioria dos vegetais analisados encaixava-se na mesma classificação como A, B ou C, de acordo com o teor de carboidratos. Na análise da adequação desta classificação para utilização no sistema AQNS, observou-se, contudo, que alguns vegetais do mesmo grupo não apresentaram as mesmas características quanto ao modo de preparo. Por exemplo, a abobrinha e o espinafre fazem parte do mesmo grupo (Grupo A), possuem o mesmo pigmento, mas possuem estrutura vegetal diferente, o que altera as técnicas de preparo e o tempo de cocção. Outro exemplo é o repolho-roxo e as abóboras (Grupo B), que apresentam diferentes pigmentos e estruturas vegetais. Ao analisar essa classificação, observou-se, como aspecto positivo, que ela apresenta grupos de vegetais com composição nutricional semelhante. Entretanto, uma deficiência para utilizá-la no sistema AQNS seria a consistência dos vegetais componentes dos grupos, devido às diferenças de estrutura dos tecidos celulares.

\section{Concepção de um novo referencial da classificação dos vegetais para a aplicação do sistema AQNS}

Nessa etapa do trabalho, buscou-se o cruzamento das classificações expostas anteriormente, utilizando-se os 99 alimentos trabalhados como no exemplo do Quadro 1 para abóboras e realizando-se os cruzamentos conforme já explicitado.

A classificação considerada adequada ao sistema AQNS originou-se da utilização da classi- 
ficação pela parte botânica, associada à classificação pelo teor de carboidratos, atualizada segundo a Tabela Brasileira de Composição de Alimentos ${ }^{19}$. Além dessas duas classificações, quando necessário, adequaram-se os grupos de acordo com as características de processamento e utilização dos vegetais em UPR.

Sendo assim, a classificação proposta para implantação do sistema AQNS em preparações de vegetais conta com sete grupos, conforme Figura 2.

- Grupo 1 - Flores com 5\% de carboidratos: brócolis, brócolis chinês, couve-flor e alcachofra.

- Grupo 2 - Folhas, caules e brotos com $5 \%$ de carboidratos: acelga, aipo ou salsão, alho-poró, aspargo, couve-chinesa, couve-debruxelas, couve-manteiga, espinafre, mostarda crespa e lisa, repolho e repolho-roxo, agrião, alfaces americana, crespa, lisa, romana e roxa, almeirão roxo, almeirão ou radite, broto de alfafa e de feijão, chicória, endívia, escarola e rúcula. Ressalte-se que o repolho-roxo é classificado com 10\% de carboidratos pela Tabela TACO, entretanto, manteve-se no mesmo grupo pelas características de preparo.

- Grupo 3 - Frutos, raízes, tubérculos, sementes e fungos com 5\% de carboidratos: abóbora-butternut, abóbora-menina-brasileira, abóbora-mogango, abóbora-moranga, abóbora- moranga-cabotiá, abóbora-moranga-jacaré, abóbora-de-pescoço, abóbora-tetsukabuto, abobrinha, abobrinha paulista, berinjela, chuchu, cogumelos, ervilha, ervilha-torta, fava, jiló, maxixe, nabo, pepino, pimentão amarelo, verde e vermelho, quiabo, rabanete, tomate e vagem. Nesse grupo, a abóbora moranga-cabotiá é classificada com 10\% de carboidratos pela Tabela TACO, entretanto manteve-se no mesmo grupo pelas

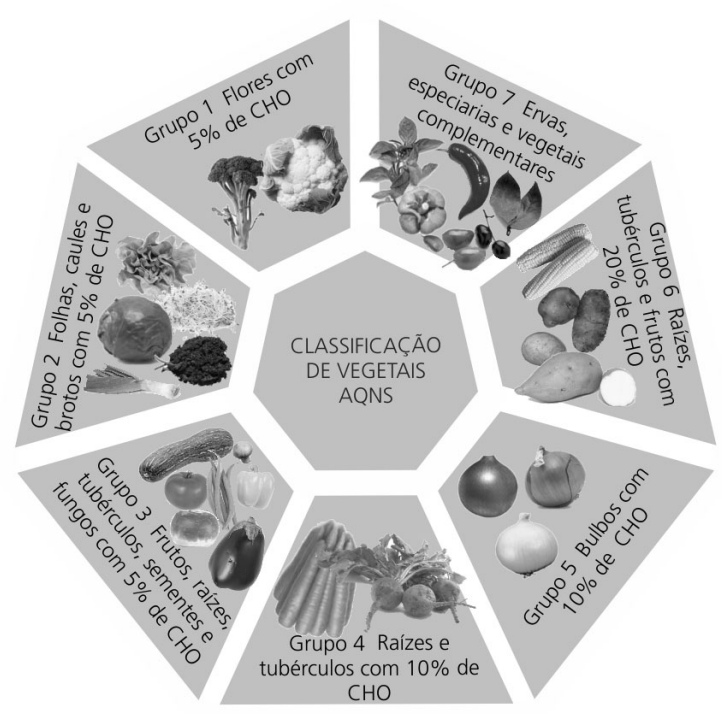

Figura 2. Roda dos vegetais AQNS.

Nota: Representação esquemática da Classificação de vegetais do sistema de avaliação da qualidade nutricional e sensorial de refeições.

AQNS: avaliação da qualidade nutricional e sensorial de refeições.

Quadro 1. Quadro com exemplo das classificações de vegetais disponíveis, utilizando abóboras.

\begin{tabular}{|c|c|c|c|c|c|c|c|}
\hline Nome português & $\begin{array}{c}\text { Nome } \\
\text { botânico }\end{array}$ & Família & $\begin{array}{c}\text { Parte } \\
\text { botânica }\end{array}$ & $\begin{array}{c}\text { Teor } \\
\text { de } \mathrm{CHO} \\
\end{array}$ & $\begin{array}{c}\text { Teor de } \mathrm{CHO} \\
\mathrm{CHO} / 100 \mathrm{~g}\end{array}$ & Pigmentos & 5 ao dia \\
\hline Abóbora-butternut & $\begin{array}{l}\text { Cucurbita } \\
\text { moschata }\end{array}$ & Cucurbitaceae & Fruto & $\begin{array}{c}\text { Grupo } \\
\text { B - } 10 \%\end{array}$ & $\begin{array}{c}\text { Grupo } \\
\text { A - } 5 \% 3 g\end{array}$ & $\begin{array}{c}\text { Carotenóide, } \\
\text { Clorofila }\end{array}$ & Amarelo \\
\hline Abóbora-menina-brasileira & $\begin{array}{l}\text { Cucurbita } \\
\text { moschata }\end{array}$ & Cucurbitaceae & Fruto & $\begin{array}{c}\text { Grupo } \\
\text { B - } 10 \%\end{array}$ & $\begin{array}{c}\text { Grupo } \\
\text { A - } 5 \% 3 g\end{array}$ & $\begin{array}{c}\text { Carotenóide, } \\
\text { Clorofila }\end{array}$ & Amarelo \\
\hline Abóbora-mogango & $\begin{array}{l}\text { Cucurbita } \\
\text { pepo }\end{array}$ & Cucurbitaceae & Fruto & $\begin{array}{c}\text { Grupo } \\
\text { B - } 10 \%\end{array}$ & $\begin{array}{c}\text { Grupo } \\
\text { A - } 5 \% 3 g\end{array}$ & $\begin{array}{c}\text { Carotenóide, } \\
\text { Clorofila }\end{array}$ & Amarelo \\
\hline Abóbora-moranga & $\begin{array}{l}\text { Cucurbita } \\
\text { máxima }\end{array}$ & Cucurbitaceae & Fruto & $\begin{array}{c}\text { Grupo } \\
\text { B - } 10 \%\end{array}$ & $\begin{array}{c}\text { Grupo } \\
\text { A - } 5 \% 3 g\end{array}$ & $\begin{array}{c}\text { Carotenóide, } \\
\text { Clorofila }\end{array}$ & Amarelo \\
\hline Abóbora-moranga cabotiá & $\begin{array}{l}\text { Cucurbita } \\
\text { pepo }\end{array}$ & Cucurbitaceae & Fruto & $\begin{array}{l}\text { Grupo } \\
\text { B -10\% }\end{array}$ & $\begin{array}{c}\text { Grupo } \\
\text { B - 10\%8g }\end{array}$ & $\begin{array}{c}\text { Carotenóide, } \\
\text { Clorofila }\end{array}$ & Amarelo \\
\hline Abóbora-moranga-jacaré & $\begin{array}{l}\text { Cucurbita } \\
\text { máxima }\end{array}$ & Cucurbitaceae & Fruto & $\begin{array}{c}\text { Grupo } \\
\text { B - 10\% }\end{array}$ & $\begin{array}{c}\text { Grupo } \\
\text { A - } 5 \% 3 g\end{array}$ & $\begin{array}{c}\text { Carotenóide, } \\
\text { Clorofila }\end{array}$ & Amarelo \\
\hline
\end{tabular}


características de preparo. Igualmente, a ervilha, a ervilha-torta, a fava e a vagem são classificadas como sementes, mas optou-se pela classificação de fruto por se adequar aos demais vegetais do grupo.

- Grupo 4 - Raízes e tubérculos com 10\% de carboidratos: cenoura e beterraba.

- Grupo 5 - Bulbos com 10\% de carboidratos: cebola, cebola roxa, cebola vermelha e chalota.

- Grupo 6 - Raízes, tubérculos e frutos com $20 \%$ de carboidratos: aipim ou mandioca, batata-baroa e batata-baroa branca, batata-doce, batata-doce branca e roxa, batata-inglesa, cará, inhame e milho verde.

- Grupo 7 - Ervas, especiarias e vegetais complementares: alho e alho roxo, azeitona verde e preta, açafrão, alecrim, alfavaca ou basilicão, cebolinha verde, coentro, cominho, cúrcuma ou açafrão-da-Índia, endro ou aneto, erva doce ou anis, estragão, gengibre, hortelã ou hortelã-pimenta, louro, manjericão, manjerona, noz-moscada, orégano, pimenta, pimenta-do-reino, salsa, sálvia, timo, tomilho ou segurelha. Esse grupo é utilizado para conferir sabor, aroma e componentes nutricionais. Entretanto, como são utilizados em pequena quantidade, foram agrupados dessa maneira.

\section{CONSIDERAÇÕ ES FIN A IS}

Foram pesquisados 99 vegetais e divididos em sete grupos. Para cada vegetal, compilaram-se informações quanto à família e parte botânica, pigmentos, principais componentes nutricionais, compostos bioativos, componentes antinutricionais, teor de carboidratos e forma de utilização em UPR. A classificação proposta compõe-se dos grupos: Flores com 5\% Carboidrato (CHO) (4 vegetais); Folhas, caules e brotos com 5\% CHO (26 vegetais); Frutos, raízes, tubérculos, sementes e fungos com 5\% CHO (27 vegetais); Raízes e tubérculos com $10 \% \mathrm{CHO}$ (2 vegetais); Bulbos com
$10 \%$ CHO (4 vegetais); Raízes, tubérculos e frutos com 20\% CHO (10 vegetais); Ervas, especiarias e vegetais complementares (26 vegetais).

Evidencia-se que o controle da interação entre aspectos nutricionais, sensoriais e de técnicas de processamento desde a aquisição até a distribuição do alimento pronto poderão, potencialmente, proporcionar alimentos mais saudáveis, pois, durante essas etapas, os nutrientes tradicionais e os não tradicionais podem ser preservados, ter a sua biodisponibilidade acentuada ou minimizadas as suas perdas. Além disso, o controle da qualidade sensorial dos alimentos influencia a escolha e consumo dos alimentos prontos.

No decorrer do estudo, conforme discutido, algumas lacunas foram observadas na literatura científica que podem ser alvo de aprofundamentos futuros para aperfeiçoamento da classificação aqui concebida. Por exemplo, no que diz respeito aos compostos bioativos e aos componentes antinutricionais, observou-se que as pesquisas são recentes e vários conceitos ainda não estão consolidados. Então, considerando também a evolução natural da ciência, sempre há a possibilidade de incremento da classificação ora apresentada com outros compostos ou outras funções para esses compostos, bem como outros componentes antinutricionais.

Outra dificuldade do presente estudo está relacionada com a definição dos critérios de qualidade nutricional e sensorial. Cabe ressaltar que se observou certa deficiência de estudos que explorem esses aspectos relacionados às técnicas de preparo.

A intenção é que essa classificação sirva como referência, tanto para o desenvolvimento do sistema, quanto para futuras pesquisas, englobando as características nutricionais, sensoriais e de técnicas de processamento. Outras possibilidades de utilização da classificação estruturada seriam: a utilização para classificação de vegetais em inquéritos alimentares, na elaboração de dietas e cardápios nas diversas áreas da nutrição; valer-se da classificação como instrumento para auxiliar nas boas práticas de produção de re- 
feições; além de poder constituir-se num recurso prático para os profissionais da área para consulta diária e desenvolvimento de novas pesquisas. Para tal, elaborou-se uma tabela para consulta rápida, onde constam várias informações, quais sejam: figura do vegetal, nome em português, inglês e francês, nome e família botânica, parte botânica comestível, teor de carboidratos, pigmentos, classificação pelo Programa 5 ao dia, 5 a day e My Pyramid, principais componentes nutricionais, principais compostos bioativos e componentes antinutricionais.

Salienta-se, como já discutido, que como as recomendações de consumo diário de vegetais são cada vez mais enfatizadas, a utilização de uma classificação como esta pode ser vislumbrada para além do sistema AQNS, como citado acima.

\section{COLABORADORES}

L.C. BORJES participou da elaboração do projeto de pesquisa, da coleta e da análise dos dados, da discussão dos resultados e da redação do artigo. S.B. CAVALLI e R.P.C. PROENÇA participaram da elaboração do projeto de pesquisa, da análise dos dados, da discussão dos resultados e da redação do artigo.

\section{REFERÊ N CIAS}

1. Food and Agriculture Organization. Rome Declaration on World Food Security and World Food Summit Plan of Action. Rome: FAO; 1996 [cited 2006 Dec 10]. Available from: <http:// WWW.fao.org/DOCREP/003/W3613E/W36 13E00.HTM>.

2. Brasil. Ministério da Saúde. Portaria $n^{\circ} 710$, de 10 de junho de 1999. Aprova a política nacional de alimentação e nutrição. Brasília: MS; 1999.

3. World Health Organization. Global strategy on diet, physical activity and health. Rome; 2004 [cited 2007 Feb 25]. Available from: <http://www.who. int/gb/ebwha/pdf-files/WHA57/A57_R17-en.pdf>.

4. Brasil. Ministério do Desenvolvimento Social e Combate à Fome. Lei n 11.346, de 15 de setembro de 2006. Cria o sistema nacional de segurança alimentar e nutricional. Diário Oficial da União. 2006; 18 set; Seção 1.
5. Proença RPC, Sousa AA, Veiros MB, Hering B. Qualidade nutricional e sensorial na produção de refeições. Florianópolis: UFSC; 2005. Série Nutrição.

6. Hering B, Proença RPC, Sousa AA, Veiros MB. Evaluation of nutritional and sensorial quality in meal production - NSQE system. J Foodservice. 2006; 17(4):173-81.

7. Dutra-de-Oliveira JE, Marchini JS. Ciências nutricionais. São Paulo: Sarvier; 1998.

8. Bellisle F, Guy-Grand B, Le Magnen J. Chewing and swallowing as indices of the stimulation to eat during meals in humans: effects revealed by the edogram method and video recordings. Neurosc Biobehav Rev. 2000; 24(2):223-8.

9. Alvarez P, Blanco MA. Reliability of the sensory analysis data of a panel of tasters. J Sci Food Agric. 2000; 80(3):409-18.

10. Ornellas LH. Técnica dietética: seleção e preparo de alimentos. $8^{a}$ ed. São Paulo: Atheneu; 2006.

11. Akutsu RC, Botelho RA, Camargo EB, Sávio KEO, Araújo WC. A ficha técnica de preparação como instrumento de qualidade na produção de refeições. Rev Nutr. 2005; 18(2):277-9. doi: 10.1590/S1 415-52732005000200012.

12. Silva Júnior EA. Manual de controle higiênico-sanitário em alimentos. $6^{a}$ ed. São Paulo: Varela; 2005.

13. International Commission on Microbiological Specifications for Foods. APPCC na qualidade e segurança microbiológica de alimentos. São Paulo: Varela; 1997.

14. Correa MP. Dicionário das plantas úteis do Brasil e das exóticas cultivadas. Rio de Janeiro: Imprensa Nacional; 1926. v.6.

15. Weberling F, Schwantes HO. Taxionomia vegetal. São Paulo: EPU; 1986.

16. Gemtchújnicov ID. Manual de taxonomia vegetal. São Paulo: Agronômica Ceres; 1976.

17. Joly $A B$. Botânica: introdução à taxonomia vegetal. $11^{\text {a }}$ ed. São Paulo: Companhia Editora Nacional; 1993.

18. De Angelis RC. A importância dos alimentos vegetais na proteção da saúde: fisiologia da nutrição protetora e preventiva de enfermidades degenerativas. $2^{a}$ ed. São Paulo: Atheneu; 2005.

19. Universidade Estadual de Campinas. Núcleo de Estudo e Pesquisa em Alimentação. Tabela brasileira de composição de alimentos: versão 2. Campinas: Unicamp; 2006.

20. MacDougall DB. Colour in food: improving quality. Cambridge (England): Woodhead Publishing; 2002 [cited 2005 Nov 2]. Available from: <http://www. foodnetbase.com>. 
654 | L.C. BORJES et al.

21. Instituto Brasileiro de Orientação Alimentar. 5 ao dia [acesso 2005 maio 27]. Disponível em: <http:// www.5aodia.com.br>.

22. World Health Organization. Codex Alimentarius 30th Session of the Codex Alimentarius Commission. Geneva; 2007 [cited 2008 Jan 31]. Available from: <http://www.codexalimentarius. net/gsfaonline/ foods/index.html?id=83>.

23. United States Department of Agriculture. My pyramid. 2005 [cited 2005 May 27]. Available from: $<$ http://www.mypyramid.gov>.

24. Ferreira $A B H$. Novo dicionário da língua portuguesa. $3^{a}$ ed. Curitiba: Positivo; 2004.
25. Philippi ST. Nutrição e técnica dietética. $2^{a}$ ed. Barueri: Manole; 2006.

26. Maincent M. Technologie culinaire: personnel, equipement, material, produits, hygiene et securite. Editeur: Bpi; 1997.

27. Produce for better health foundation. 5 a day the color way. 1991 [cited 2005 May 27]. Available from: <http://www.5aday.com/index.php>.

Recebido em: 23/10/2008

Versão final reapresentada em: 17/8/2009 Aprovado em: 18/3/2010 